\title{
Establishment of Wellbore Temperature Field During High Temperature and High Pressure Well Cementing in Western South China Sea
}

\author{
Pan Rui ${ }^{1}$, Guan Zhichuan ${ }^{1}$, Luo Ming ${ }^{2}$, Ma Xianming ${ }^{1}$, Wang Kai ${ }^{1}$, Wang Bin ${ }^{1}$ \\ ${ }^{1}$ School of Petroleum Engineering, China University of Petroleum (East China), Qingdao, China \\ ${ }^{2}$ Zhanjiang Branch, CNOOC China Limited, Zhanjiang, China
}

\section{Email address:}

pr359952060@163.com (Guan Zhichuan)

\section{To cite this article:}

Pan Rui, Guan Zhichuan, Luo Ming, Ma Xianming, Wang Kai, Wang Bin. Establishment of Wellbore Temperature Field During High Temperature and High Pressure Well Cementing in Western South China Sea. Science Discovery. Vol. 7, No. 1, 2019, pp. 39-44. doi: $10.11648 /$ j.sd.20190701.19

Received: January 18, 2019; Accepted: April 3, 2019; Published: April 28, 2019

\begin{abstract}
The western part of south China sea is characterized by high geothermal gradient, abnormal overpressure development and narrow formation pressure window in the deep part of the basin. In the process of cementing, the annulus has high pressure consumption, high risk of leakage, and significant influence of temperature on the rheology and hydration of cement slurry. In order to accurately determine the rheological and rheological parameters of cement slurry and prevent possible well leakage, overflow and even blowout accidents in the process of cementing, it is necessary to establish a wellbore temperature field model in the process of cementing. Based on the data of the actual drilling well, the variation of wellbore temperature under different pump displacement and circulation time is analyzed. The results show that the annular temperature is larger than the annular temperature at the beginning, and the annular temperature is larger than the annular temperature with the increase of depth. With the increase of displacement, the bottom hole temperature decreases and the outlet temperature increases. With the extension of the cycle time, the bottom hole temperature decreases and the outlet temperature increases. When the cycle time reaches a certain value, the bottom hole and outlet temperature remain basically unchanged.
\end{abstract}

Keywords: Western South China Sea, The Cementing, Risk of Leakage, Cement Slurry, Wellbore Temperature Field Model

\section{南海西部高温高压井注水泥过程中井筒温度场的建立}

\author{
潘瑞1，管志川 ${ }^{1}$ ，罗鸣 ${ }^{2}$, 马贤明 ${ }^{1}$, 王凯 ${ }^{1}$, 王斌 ${ }^{1}$ \\ 1 中国石油大学(华东)石油工程学院, 青岛, 中国 \\ 2中海石油(中国)有限公司湛江分公司, 湛江, 中国
}

\section{邮箱}

pr359952060@163.com（管志川）

\begin{abstract}
摘要: 中国南海西部地区具有高地温梯度, 异常超压发育以及地层压力窗口狭窄等特点。在固井过程中, 环空压耗大, 漏失风险高, 温度对水泥浆的流变性和水化有十分显着的影响。为了准确确定水泥浆的流变性和流变学参数, 防止固 井过程中可能发生的井漏、甚至气窝等问题, 有必要建立固井过程井筒温度场模型。以某实钻井为例, 分析了不同原 排量和顶替时间下井筒温度的变化规律。结果表明: 管内温度开始是大于环空温度, 随深度增加环空温度大于管内温 度。随着排量的增大, 井底温度降低, 出口温度升高。随着顶替时间的延长, 井底温度降低, 出口温度升高, 顶替时 间到达一定值时井底和出口温度基本不变。
\end{abstract}


关键词: 南海西部, 固井, 漏失风险, 水泥浆, 井筒温度场模型

\section{1. 引言}

海上钻井作业不同于陆地, 由于海水段的存在，一方 面, 海水相较于陆地而言上覆压力有所亏欠, 导致地层深 处破裂压力和地层压力窗口狭窄; 另一方面, 海水的温度 梯度与地温梯度呈现相反的增长趋势。另外, 南海西部莺 歌海盆地底辟且热流体活动强烈, 属于新生代高温高压盆 地[1], 这使得深处地层压力窗口变得更加狭窄。并且温度 在很大程度上会影响流体的密度以及流变性[2-5], 使得环 空压力不断波动, 在实际钻进和注水泥施工过程中，易发 生井漏。

海上钻井作业期间的井筒温度是海上钻井设计和安 全钻井的重要基础数据[6]。目前, 国内外学者大多对钻进 过程中的温度场展开研究(流体仅为单介质钻井液)。但是, 对于注水泥施工存在多重介质的井筒温度场研究不足。就 海上注水泥过程中井筒温度场而言, 海水段的传热与地层 处的传热存在很大差异, 海水段往往以对流换热为主; 而 地层段由于固井设计水泥浆并未返至泥线, 环空中存在钻 井液, 此时, 地层传热更为复杂。加之, 注水泥是一个多 种流体并存的过程, 并且水泥浆具有水化放热性质, 这使 得研究该工况下的井筒温度场变得十分困难, 笔者不得不 对所研究的问题加以简化。综上所述, 对莺-琼盆地注水 泥施工井筒温度场的研究是十分有必要的。

现今, 安全、高效、经济地开发海上能源是作为当前 和以后的发展趋势, 那么, 建立井筒温度场又是关键性的 一环。虽然, 现在的技术能够实测温度, 但由于费用过于 昂贵, 不可能每口井都利用仪器实测。加之, 实测温度仅 在钻进过程, 注水泥过程无法实测, 所以, 对井筒温度建 立模型是必须的。当前, 国内外许多学者都对井筒温度场 进行了建立, 其研究方法不外乎稳态法、半稳态法以及全 瞬态法, 求解过程也不外乎直接法(即可以直接求得温度 方程)和间接法(必须通过数值法进行求取)。其中，较为经 典的是, Ramey[7]提出的半稳态模型和Raymond[8]提出的 全瞬态模型; 国内方面, 宋洵成[9]对深水井进行研究并提 出的全瞬态模型, 以及杨谋 [10]针对一口深井提出的瞬态 传热模型等。基于以上研究, 笔者对固井施工井筒温度场 展开研究。

\section{2. 井筒传热的物理模型}

由海上注水泥井筒传热特点将井筒温度场分为海水 段和地层段, 以7in尾管固井为例, 并对井筒传热物理模 型进行划分(如图1所示): $5.5 \mathrm{in}$ 送入钻杆内流区 $\left(0 \leq \mathrm{r} \leq \mathrm{r}_{\mathrm{pi}}\right)$, 尾管内流区 $\left(0 \leq \mathrm{r} \leq \mathrm{r}_{\mathrm{lbi}}\right)$, 水泥浆从钻井平台通过送入钻杆至 井底; 送入管柱本体区 $\left(\mathrm{r}_{\mathrm{p} i} \leq \mathrm{r} \leq \mathrm{r}_{\mathrm{po}}\right)$; 隔水管本体区 $\left(\mathrm{r}_{\mathrm{r}} \leq \mathrm{r} \leq \mathrm{r}_{\mathrm{ro}}\right)$; 井壁区 $\left(r=r_{\mathrm{co}} / \mathrm{r}_{\mathrm{lo}}\right)$; 裸眼环空流区 $\left(\mathrm{r}_{\mathrm{lbo}} \leq \mathrm{r} \leq \mathrm{r}_{\mathrm{i}}\right)$, 送入钻杆与套 管环空流区 $\left(\mathrm{r}_{\mathrm{po}} \leq \mathrm{r} \leq \mathrm{r}_{\mathrm{c} i}\right)$, 送入管柱与隔水管环空流区 $\left(r_{\mathrm{po}} \leq \mathrm{r} \leq \mathrm{r}_{\mathrm{r}}\right)$ 。海水段的传热过程在径向上: 送入管柱与内外 两侧流体的对流换热, 隔水管与内外两侧流体的对流换热,
其中, 与海水段接触的为强制对流换热, 以及海水在径向 上的热传递。轴向上: 送入管柱内外流体流动过程中的热 交换以及管柱本体的导热。地层段的传热过程在径向上: 送入管柱与内外两侧流体的对流换热, 9.625in套管与内侧 流体之间的对流换热, 外侧与地层之间的导热, 外侧与未 封固地层之间的对流换热, 尾管内外两侧的对流换热和裸 眼环空处环空流体与地层之间的对流换热, 以及地层径向 上的热传递。轴向上: 流体在送入管柱、套管柱内和套管 环空内流动的热交换以及管柱本体的导热等。

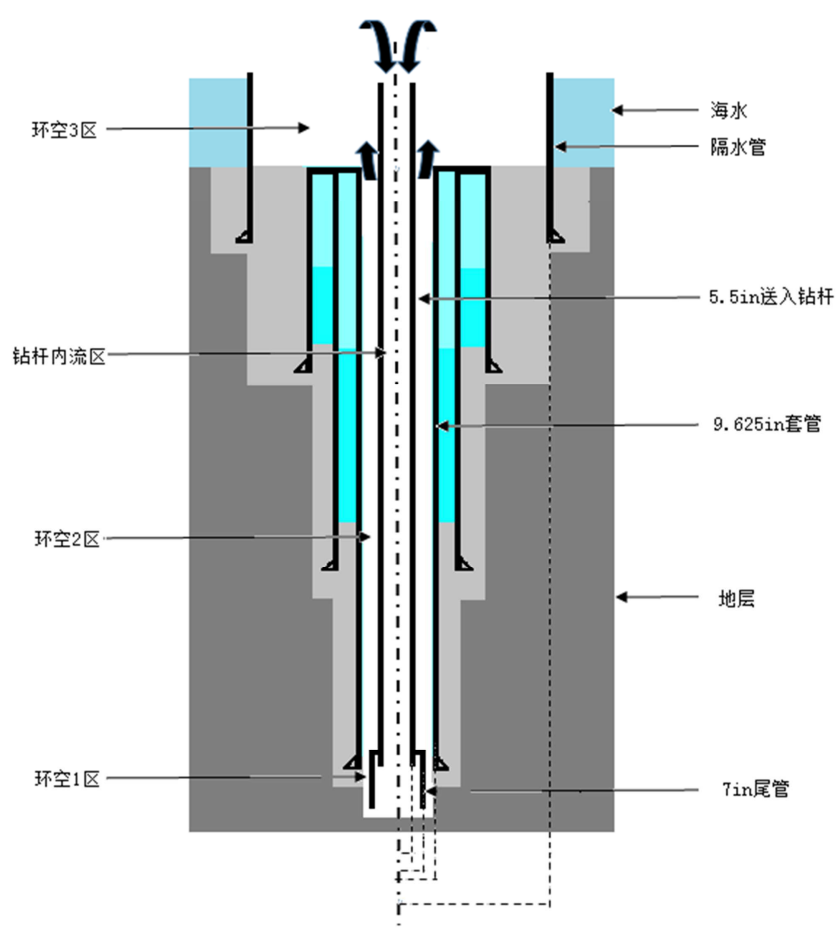

图1 井筒传热物理模型。

\section{3. 井筒传热的数学模型}

\section{1. 模型的基本假设和基本原理}

流体在井筒内流动主要是在送入管柱内、套管内以及 管外的环空中流动, 由于流体的流变性不同, 所以相应的 就会产生不同的流型, 对于钻井液和水泥浆而言, 宾汉 模式、幂律模式以及赫-巴模式拟合的效果会更好[12]。另 外, 根据流体在井筒中流动状态呈现出层流和湍流, 流体 的流态受到流量、流道等的制约, 其将会对传热造成影响。

注水泥施工牵扯到钻井液、水泥浆等多种非牛顿流体, 其流变模式和流态在井筒循环系统中不断地发生变化, 并 且受到诸如排量、井筒几何形状、井筒尺寸大小以及循环 时间等诸多因素的干扰, 相应地会很大程度上影响井筒温 度场的分布, 另外, 由于水泥浆的水化放热作用对井筒温 度场也具有一定程度上的影响。由于该过程的复杂性和困 难性, 现阶段, 国内外学者对注水泥过程中井筒温度场的 研究存在空缺。为满足实际工程需要, 并考虑到模型的复 
杂性和困难程度, 笔者现做出如下的假设, 使模型的计算 具有可行性以及达到适合的工程预测精度。

(1) 裸眼井段自始至终无扩、缩径;

(2) 不考虑水泥浆的水化放热;

(3) 送入钻杆与井眼轴线同心且不发生偏移, 尾管在井 下居中;

(4) 管内、环空中的流体性质不发生变化, 视为各向同 性的液体;

(5) 认为地层、海水的热物性参数均不发生变化为一定 值;

(6) 水泥浆、钻井液等注水泥过程中的流体, 其热物性 参数为常值, 且流体不可压缩;

(7) 地层视为均质地层, 并相对于井眼轴线呈对称分布, 且地层仅存在导热, 不考虑地层中的热源, 认为地 层、海水温度始终不发生变化;

(8) 管内、环空中认为仅存在轴向上的一维传热, 在径 向上没有温度变化, 即不存在径向温度梯度; 钻杆、 尾管管体则为径向和轴向的二维传热。

(9) 考虑流体循环流动时摩擦生热并作为内热源计算。 井筒传热模型的基础是运用热力学第一定律(能量守 恒定律), 把所研究的微元控制体视为一个热力学系统, 该系统内能的增量等于外界向它传递的热量与外界对它 所做的功之和。

$$
\Delta \mathrm{U}=\mathrm{W}+\mathrm{Q}
$$

\section{2. 井筒循环温度场模型的控制方程}

经过文献分析，笔者类比于文献[9-11]中建立的温度 场控制方程。根据之前的假设以及热力学第一定律、传热 学基本原理为基础, 分别为管柱内流、管柱本体、环空流 列控制方程，可得到全井段井筒温度场模型的控制方程。 由于海水段和地层段存在诸如温度分布、传热特点等的差 异, 因此分别对这两段建立井筒温度场的控制方程。

\subsection{1. 海水段的控制方程}

1)送入钻杆内流体循环的控制方程

对于送入钻杆内流体而言, 任取高为 $\mathrm{dz}$, 半径为钻杆 内径 $r_{\mathrm{pi}}$ 的微元控制体, 在 $\mathrm{dt}$ 时间内的传热有: 轴向上, 流 入与流出控制体的热量, 由比内能公式得到; 径向上, 钻 杆内壁面与微元控制体的对流换热, 由牛顿冷却公式得到; 以及在流体流动过程中的摩擦做功形成的内热源。经整理 可得:

$$
\begin{aligned}
& Q_{f p}-\frac{\partial\left(\rho_{l} q c_{l} T_{p}\right)}{\partial z}-2 \pi r_{p i} h_{p i}\left(T_{p}-T_{w}\right) \\
& =\frac{\partial\left(A_{p i} \rho_{l} c_{l} T_{p}\right)}{\partial t}
\end{aligned}
$$

2)送入钻杆管体的控制方程

对于送入钻杆管体而言, 同样任取高为 $d z$ 的微元控制 体, 在 $d t$ 时间内的传热有: 轴向上, 管体内部的导热, 由 傅里叶导热定律得出; 径向上, 管体内外壁面与两侧流体 之间的对流换热, 由牛顿冷却公式得到。根据热力学第一
定律可阐释成: 轴向上和径向上在 $\mathrm{dt}$ 时间内净流入控制体 的热能全部转化为内能的增量。经整理可得:

$$
\begin{aligned}
& k_{w} \frac{\partial^{2} T_{w}}{\partial z^{2}}+\frac{2 r_{p o} h_{p o}\left(T_{a}-T_{w}\right)}{r_{p o}^{2}-r_{p i}^{2}} \\
& +\frac{2 r_{p i} h_{p i}\left(T_{p}-T_{w}\right)}{r_{p o}^{2}-r_{p i}^{2}}=\frac{\partial\left(\rho_{w} c_{w} T_{w}\right)}{\partial t}
\end{aligned}
$$

3)钻杆与隔水管之间环空3的控制方程

对于该环空流体而言, 也任取高为 $d z$ 的微元控制体, 在 $\mathrm{dt}$ 时间内的传热有: 轴向上, 流入与流出控制体的热量, 由比内能公式得到; 径向上, 钻杆外壁面和隔水管内壁面 与微元控制体的对流换热, 由牛顿冷却公式得到; 以及在 流体流动过程中的摩擦做功形成的内热源。经整理可得:

$$
\begin{gathered}
Q_{f a 3}+\frac{\partial\left(\rho_{l} q c_{l} T_{a}\right)}{\partial z}+2 \pi r_{r i} h_{s}\left(T_{s}-T_{a}\right) \\
+2 \pi r_{p o} h_{p o}\left(T_{w}-T_{a}\right)=\frac{\partial\left(A_{a 3} \rho_{l} c_{l} T_{a}\right)}{\partial t}
\end{gathered}
$$

\subsection{2. 地层段的控制方程}

地层段的控制方程与海水段的控制方程在很大程度 上有相似之处, 二者的差异主要在环空处的控制方程。

1)送入钻杆内流体循环的控制方程

传热特点同海水段送入钻杆内流体的传热, 控制方程 同式(2)。

2)尾管内流体循环的控制方程

传热特点同海水段送入钻杆内流体的传热, 只不过介 质由钻杆变成尾管，其控制方程为:

$$
\begin{aligned}
& Q_{f p}-\frac{\partial\left(\rho_{l} q c_{l} T_{p}\right)}{\partial z}-2 \pi r_{l b i} h_{l b i}\left(T_{p}-T_{w}\right) \\
& =\frac{\partial\left(A_{l b i} \rho_{l} c_{l} T_{p}\right)}{\partial t}
\end{aligned}
$$

3)送入钻杆管体的控制方程

传热特点同海水段送入钻杆管体的传热, 控制方程同 式(3)。

4)尾管管体的控制方程

传热特点同送入钻杆管体的传热, 根据送入钻杆管体 的控制方程, 经整理可得:

$$
\begin{aligned}
& k_{w} \frac{\partial^{2} T_{w}}{\partial z^{2}}+\frac{2 r_{l b o} h_{l b o}\left(T_{a}-T_{w}\right)}{r_{l b o}^{2}-r_{l b i}^{2}} \\
& +\frac{2 r_{l b i} h_{l b i}\left(T_{p}-T_{w}\right)}{r_{l b o}^{2}-r_{l b i}^{2}}=\frac{\partial\left(\rho_{w} c_{w} T_{w}\right)}{\partial t}
\end{aligned}
$$

\section{5)钻杆与套管环空 2 的控制方程}

对于该环空流体而言, 任取高为 $\mathrm{dz}$ 的微元控制体, 在 $\mathrm{dt}$ 时间内的传热有: 轴向上, 流入与流出控制体的热量, 由比内能公式得到; 径向上, 钻杆外壁面与微元控制体的 对流换热, 由牛顿冷却公式得到, 还有地层与微元控制体 的传热(包括：地层与井壁的导热和井壁到环空的对流换 
热); 以及在流体流动过程中的摩擦做功形成的内热源。 经整理可得:

$$
\begin{aligned}
& Q_{f a 2}+\frac{\partial\left(\rho_{l} q c_{l} T_{a}\right)}{\partial z}+\frac{2 \pi r_{c i} h_{f} k_{f}}{r_{c i} h_{f} f(t)+k_{f}}\left(T_{f}-T_{a}\right) \\
& +2 \pi r_{p o} h_{p o}\left(T_{w}-T_{a}\right)=\frac{\partial\left(A_{a 2} \rho_{l} c_{l} T_{a}\right)}{\partial t}
\end{aligned}
$$

6)裸眼环空 1 的控制方程

对于该环空流体而言，任取高为 $\mathrm{dz}$ 的微元控制体，在 $\mathrm{dt}$ 时间内的传热有: 轴向上, 流入与流出控制体的热量, 由比内能公式得到; 径向上, 尾管外壁面和裸眼处地层与 微元控制体的对流换热, 由牛顿冷却公式得到; 以及在流 体流动过程中的摩擦做功形成的内热源。经整理可得:

$$
\begin{gathered}
Q_{f a 1}+\frac{\partial\left(\rho_{l} q c_{l} T_{a}\right)}{\partial z}+2 \pi r_{i} h_{f}\left(T_{f}-T_{a}\right) \\
+2 \pi r_{l b o} h_{l b o}\left(T_{w}-T_{a}\right)=\frac{\partial\left(A_{a 1} \rho_{l} c_{l} T_{a}\right)}{\partial t}
\end{gathered}
$$

式中: $\mathrm{Q}_{\mathrm{f}}$ 为流体流动时的摩擦生热量, $\mathrm{W}\left(\mathrm{Q}_{\mathrm{fp}} 、 \mathrm{Q}_{\mathrm{fa}}\right.$ 分别为管内和环空流的摩擦生热量); $\rho_{1}$ 为流体密度, $\mathrm{kg} / \mathrm{m}^{3} ; \mathrm{q}$ 为体积流量, $\mathrm{m}^{3} / \mathrm{s} ; \mathrm{c}$ 为比热容, $\mathrm{J} /\left(\mathrm{kg} \bullet{ }^{\circ} \mathrm{C}\right.$ )(其中, $\mathrm{c}_{1} 、 \mathrm{c}_{\mathrm{w}}$ 分别为流体、管材的比热容); $\mathrm{T}$ 为温度, ${ }^{\circ} \mathrm{C}$ (其中 $T_{p} 、 T_{w} 、 T_{a} 、 T_{s}$ 以及 $T_{f}$ 、分别为管内、管体、环空、海 水、地层温度); $r$ 为半径, $\mathrm{m}$ (其中, 下标pi、po、 $\mathrm{lbi}$ 、 lbo、 ri、 ci分别为送入钻杆内外径、尾管内外径、隔水 管内径、9.625in套管内径 $) ; \mathrm{k}$ 为热导率，W/(m・ $\left.{ }^{\circ} \mathrm{C}\right)\left(\mathrm{k}_{\mathrm{w}}\right.$ 、 $\mathrm{k}_{\mathrm{f}}$ 分别为管体和地层的热导率); $\mathrm{h}$ 为对流换热系数, $\mathrm{W} /\left(\mathrm{m}^{2} \cdot{ }^{\circ} \mathrm{C}\right)\left(\mathrm{h}_{\mathrm{pi}} 、 \mathrm{~h}_{\mathrm{po}} 、 \mathrm{~h}_{\mathrm{s}} 、 \mathrm{~h}_{\mathrm{lbi}} 、 \mathrm{~h}_{\mathrm{lbo}}\right.$ 及 $\mathrm{h}_{\mathrm{f}}$ 分别为流体与送入 钻杆内外壁、海水与环空流体、流体与尾管内外壁以及
流体与地层的对流换热系数 $)$; $\mathrm{A}$ 为面积, $\mathrm{m}^{2}\left(\mathrm{~A}_{\mathrm{pi}} 、 \mathrm{~A}_{\mathrm{lbi}}\right.$ 、 $\mathrm{A}_{\mathrm{a} 1} 、 \mathrm{~A}_{\mathrm{a} 2} 、 \mathrm{~A}_{\mathrm{a} 3}$ 分别为送入钻杆、尾管、环空1、环空2、 环空 3 的有效截面积)。

\section{3. 模型求解}

(1)初始、边界条件

$\mathrm{T}_{\mathrm{p}, \mathrm{j}}=\mathrm{T}_{\mathrm{w}}, \mathrm{j}=\mathrm{T}_{\mathrm{a}}, \mathrm{j}=\mathrm{T}_{\mathrm{s}}, \mathrm{j}\left(\mathrm{t}=0, \quad 0 \leq \mathrm{j} \leq \mathrm{H}_{\mathrm{s}}\right)$

$T_{p}, j=T_{w}, j=T_{a}, j=T_{f}, j\left(t=0, \quad H_{s} \leq j \leq H\right)$

入口温度: $\mathrm{T}_{p, 0}^{i}=\mathrm{T}_{\mathrm{in}}(\mathrm{i} \Delta \mathrm{t})(0 \leq \mathrm{i} \leq \mathrm{N})$

井底温度: $T_{p, H}^{n}=T_{w, H}^{n}=T_{a, H}^{n}(0 \leq \mathrm{n} \leq \mathrm{N})$

地层温度: $T_{f, j}^{n}=T_{f}(\mathrm{j})(0 \leq \mathrm{j} \leq \mathrm{H})$

(2)求解过程

对于海上温度场求解而言, 外界环境温度梯度不单一, 必须采用数值方法 [13]。首先, 利用有限差分法对控制方 程在时间和空间上进行离散, 将偏导做全隐式格式差分处 理, 获得离散方程组。其次, 将初始、边界条件做离散化 处理。最后, 将离散方程组化为代数方程组进行求解。

\section{4. 算例分析}

现对南海一口实钻探井展开分析, 井型为直井。该井 的基本参数如下: 施工平台为 $\mathrm{KT} 3$ (半潜式)钻井平台, 转 盘高 $23 \mathrm{~m}$, 海水深度 $87 \mathrm{~m}$, 井深 $4079 \mathrm{~m}$, 尾管顶深 $3924 \mathrm{~m}$, $7 \mathrm{in}$ 尾管, 上层套管 $9.625 \mathrm{in}, 30 \mathrm{in}$ 隔水管, $5.5 \mathrm{in}$ 送入钻杆, 排量 $4.0 \mathrm{bpm}(10.6 \mathrm{~L} / \mathrm{s})$, 水泥浆入口温度 $40^{\circ} \mathrm{C}$, 海表温度 $25^{\circ} \mathrm{C}$, 井底温度地层 $187^{\circ} \mathrm{C}$, 海温梯度为 $-0.0667^{\circ} \mathrm{C} / \mathrm{m}$, 地温梯度 为 $0.0423^{\circ} \mathrm{C} / \mathrm{m}$, 地层、流体等热物性参数见表 1 。

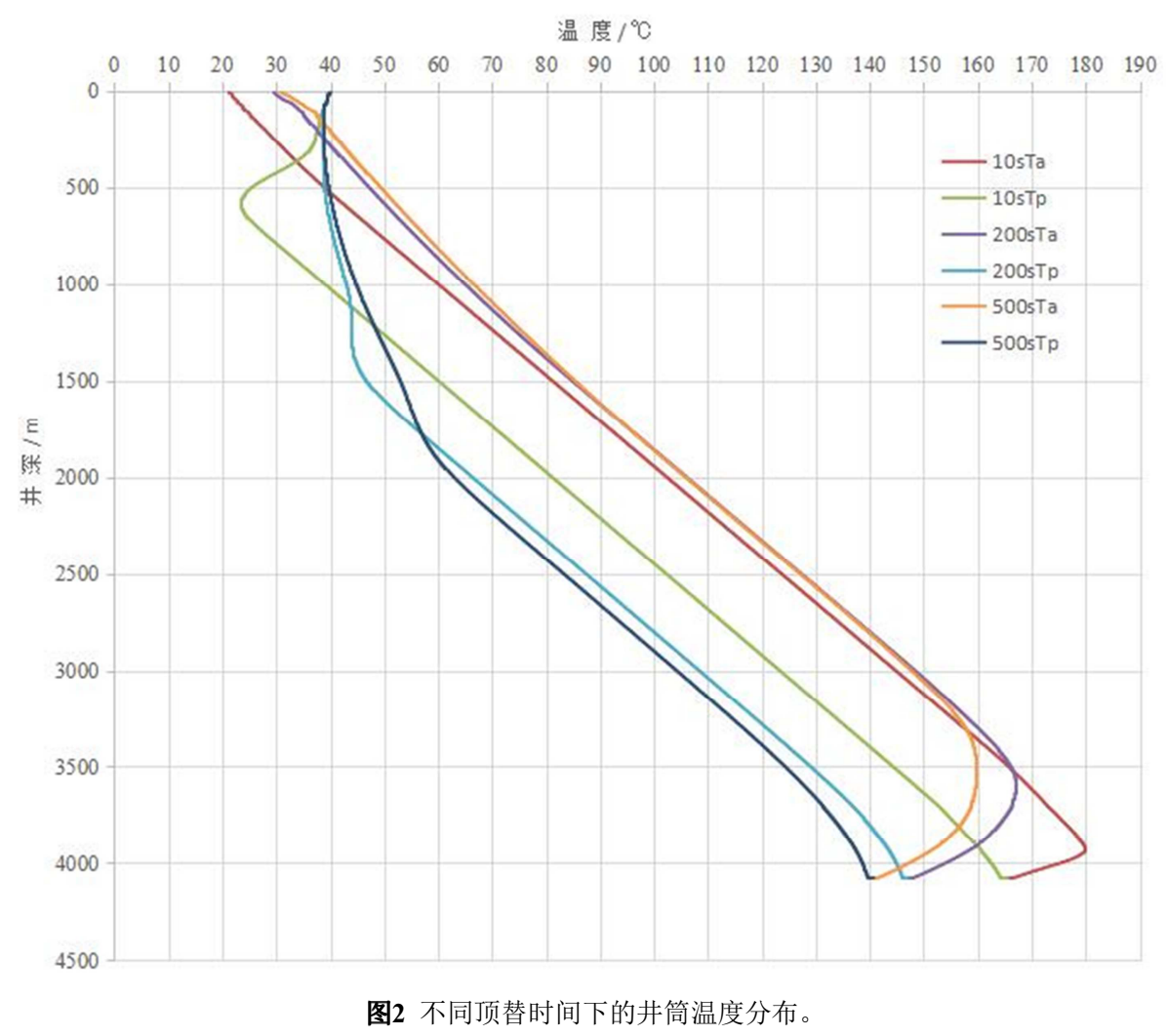




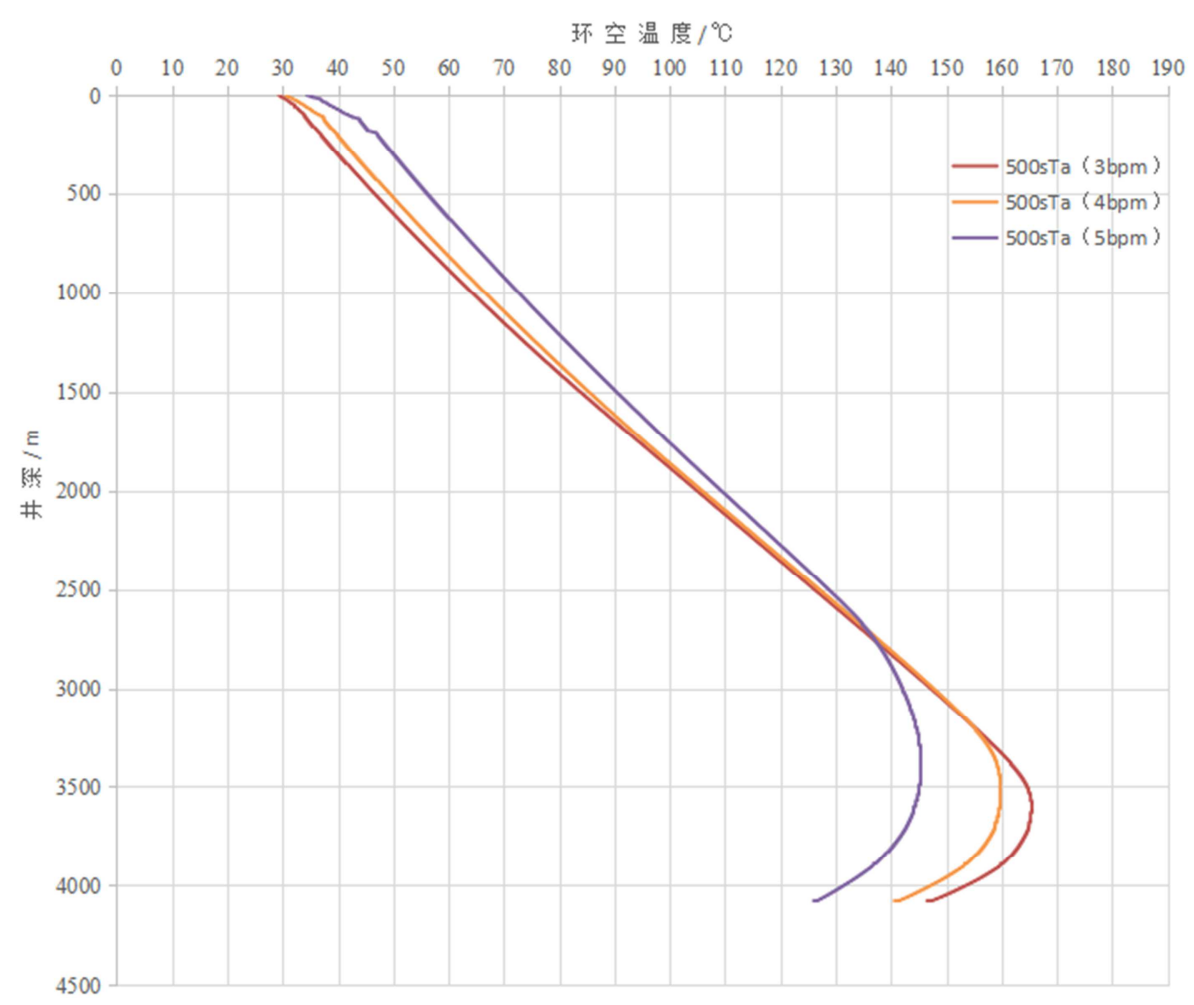

图3 不同排量下的环空温度。

表1 地层、流体等热物性参数。

\begin{tabular}{llll}
\hline 介质 & 密度 $\left(\mathbf{g} / \mathbf{c m}^{3}\right)$ & 比热 $/\left[\mathbf{J} \cdot\left(\mathbf{k g} \cdot{ }^{\circ} \mathbf{C}\right)^{-1}\right]$ & $\begin{array}{l}\text { 导热系数 } \\
/\left[\mathbf{W} \cdot\left(\mathbf{m}^{\circ}{ }^{\circ} \mathbf{C}\right)^{-1}\right]\end{array}$ \\
\hline 海水 & 1.03 & 3900 & 0.6 \\
地层 & 2.64 & 870 & 2.25 \\
钻井液 & 2.29 & 2000 & 1.75 \\
水泥浆 & 2.40 & 1650 & 2.0 \\
管柱 & 7.80 & 400 & 46.0 \\
\hline
\end{tabular}

图2给出了在排量为 $4 \mathrm{bpm}$ 下, 不同顶替时间的井筒温 度分布曲线。图中 $\mathrm{Ta}$ 为环空温度, $\mathrm{Tp}$ 为管内温度, 从图中 不难发现, 管内温度开始是大于环空温度, 随深度增加环 空温度大于管内温度; 随顶替时间的延长, 环空温度等于 管内温度的点位逐渐上移，在500s 附近基本不发生变化; 环空温度的最大值并不是在井底取得, 而是在井底以上位 置处取得, 在 $500 \mathrm{~s}$ 附近温度的最大值的位置基本不发生变 化。

图3给出了顶替时间为 $500 \mathrm{~s}$ 下，不同注入排量下的环 空温度分布曲线。随排量的增加井底温度逐渐减小, 井口 上返温度逐渐增大, 这是因为流速变大, 使得流体与地层 和海水换热的时间缩短造成的; 其最大环空温度也随排量 的增加有所上移。

\section{5. 结论}

文章中建立的注水泥井筒温度场模型, 主要解决了因 排量、注水泥顶替时间等参数引起井筒温度的变化, 进一 步填补了注水泥过程中温度场预测的空缺。若要更加精确 预测则需要考虑水泥浆在循环过程的水化放热作用。
顶替时间对井筒温度具有一定的影响, 随时间的延伸 井底和环空最大温度减小, 井口上返温度增加, 当顶替时 间到达一定的值时井筒温度基本不发生变化。

排量对环空温度的影响明显, 且随施工排量的增加, 井口上返温度增加, 井底温度和环空获得的最大温度将减 小。

\section{参考文献}

[1] 罗鸣, 韩成, 陈浩东, 等. 南海西部高温高压井堵漏技术 [J]. 石油钻采工艺，2016，38(6): 801-804。

[2] 管志川. 温度和压力对深水钻井油基钻井液液柱压力的影 响 [J]. 石油大学学报(自然科学版), 2003,27(4): 48-52。

[3] 徐璧华, 何可, 何松, 等. 低温对水泥浆流变性影响规律 分析[J]. 内蒙古石油化工, 2012,12:7-8。

[4] 王伟, 黄柏宗.高温高压下水泥浆的流变性及其模式 [J]. 油 田化学, 1994,11(1): 18-21。

[5] 王斌斌, 王瑞和. 固井水泥浆流变规律实验研究 [J]. 石油钻 采工艺，2010,32(2): 42-45。

[6] 宋洵成, 管志川. 深水钻井井筒全瞬态传热特征 [J]. 石油学 报，2011，32(4): 704-708。

[7] Ramey H J. Wellbore heat transmission [J] .Journal of Petroleum Technology, 1962, 14(4):427-435. 
[8] Raymond L R. Temperature distribution in a circulating drilling fluid [J] .Journal of Petroleum Technology, 1969, 21(3):333-341.

[9] Mou Yang, Xiaoxiao Li, Jianmin Deng, et al. Prediction of wellbore and formation temperatures during circulation and shut-in stages under kick conditions [J]. Energy, 2015, 91: 1018-1029.

[10] Xuncheng Song, Yongwang Liu , Zhichuan Guan. WHTSubmersible: a simulator for estimating transient circulation tempraure in offshore wells with the semi- submersible platform[J]. Heat Mass Transfer, 2015, 51: 1425-1435.

[11] 王博. 深水钻井环境下的井筒温度压力计算方法研究 [D]. 山东青岛: 中国石油大学(华东), 2007, 21-31。

[12] 管志川, 陈庭根. 钻井工程理论与技术(第二版)[M]. 山东 青岛: 中国石油大学, 2017:270。

[13] 高永海, 孙宝江, 王志远, 等. 深水钻探井筒温度场的计 算与分析[J]. 中国石油大学学报(自然科学版), 2008, 32(2): 58-62。 